\title{
Inhalt des Heftes 1
}

RABL, R., Beitrag zur Pathologie vom vorderen Teil des Hypothalamus . . . . . 1
NAmba, M., Cytoarchitektonische Untersuchungen am Striatum. . . . . . . . . 24
Sol,ChER, H, Über einen Fall von überstandener fötaler Kohlenoxydvergiftung. . 49
SANides, F., Untersuchungen über. die histologische Struktur des Mandelkern-
gebietes . . . . . . . . . . . . . . . . . . . . . . . . . . 56

$\mathrm{D}$

as „Journal für Hirnforschung“" wird - wie bis 1942 das , Journal für Psychologie und Neurologie" - die Forschungsergebnisse des Institutes für Hirnforschung und allgemeine Biologie in Neustadt/Schwarzwald veröffentlichen. Im Mittelpunkt der Forschungen dieses Institutes steht die Hirnanatomie, und zwar jene Teile derselben, die die wichtigsten Erkenntnisquellen für die räumlichen Beziehungen zwischen materiellem Hirngeschehen und Bewußtseinserscheinungen darstellen. Vertiefung der architektonischen Gliederung des Gehirns, Aufdeckung des anatomischen Ausdrucks individueller Besonderheiten Gesunder, Kranker und ,,zurechnungsfähiger“ Asozialer, Ausnutzung der pathologischen Anatomie für die Schaffung einer ätiologischen Klassifikation der sogenannten funktionellen Neurosen und Psychosen, Klärung der aufbauenden und reparatorischen Funktionen des metamitotischen Arbeitskernes der Nervenzellen: das sind gegenwärtig die Hauptforschungsgebiete des Institutes.

\section{Bestellungen an eine Buchhandlung erbeten}

Wenn Sie unsere Literatur nicht in ihrer Buchhandlung erhalten können oder Schwierigkeiten bei der Beschaffung haben, dann wenden Sie sich bitte an eine der nachstehenden Auslieferungsstellen oder direkt an den Verlag.

Auslieferung für die Deutsche Demokratische Republik

LKG Leipziger Kommissions- und Großbuchhandel Leipzig C1, Leninstraße 16

Auslieferung für die Bundesrepublik:

Buchhandlung Kunst und Wissen, Inhaber: Erich Bieber

Stuttgart N, Hospitalstraße 33 a

Auslieferung für das gesamte Ausland:

Deutscher Buch-Export und -Import, GmbH, Leipzig C 1, Postschließfach 276

\author{
Akademie-Verlag, \\ Berlin W 8, Mohrenstraße 39, Ruf 200386 Sammelnummer \\ Telegramm-Adresse: Akademieverlag Berlın
}

\begin{abstract}
Herausgeber und verantwortlich für den Inhalt Dr. Cécile und Prof. Oskar Vogt, Institut für Hirnforschung und allgemeine Biologie, Neustadt/Schwarzwald. Verlag. Akademie-Verlag GmbH., Berlin W 8, Mohrenstraße 39 (Fernruf : 200386); Postscheckkonto. Berlin 35021 . Bestell- und Verlagsnummer dieses Heftes: 1018/3/1. Das „Journal für Hirnforschung" erscheint in zwanglosen Heften von verschicdenem Umfang. 6 Hefte bilden einen Band. Preis je Einzelheft 12,- DM. Ein Band 72,- DM. Satz und Druck: VEB Druckbaus "Maxim Gorki", Altenburg. Veröffentlicht unter der Lizenznummer 1230 des Amtes für Literatur und Verlagswesen der Deutschen Demokratischen Republik. Printed in Germany.
\end{abstract}

